\title{
Galaxy Evolution in the Virgo Cluster
}

\section{Bernd Vollmer*}

CDS, Observatoire astronomique de Strasbourg, 11 rue de l'université, 67000 Strasbourg,

France

E-mail: bvollmereastro.u-strasbg.fr

The environment plays a key role for the evolution of galaxies residing in galaxy clusters. The detection of a large-scale, diffuse radio halo around the big elliptical galaxy M86 is reported and its possible interaction with the Virgo cluster is discussed. A galaxy entering a cluster can have three kinds of interaction: (i) an interaction with the gravitational potential of the cluster, (ii) an interaction with another galaxy, (iii) ram pressure stripping due to the galaxy's rapid motion through the hot intracluster medium. Since Virgo is the nearest cluster in the northern hemisphere, it represents an ideal place to investigate these interactions in detail. Spiral galaxies which are evolving in a cluster environment show decreased gas content and a reduced star formation rate. This is in general due to a truncation of their gas and star forming disks, whereas the inner disks have normal gas surface densities and star formation rates with respect to field galaxies of the same size and Hubble type. We have studied 6 individual Virgo cluster spiral galaxies that experienced or experience an ISM-ICM interaction, a galaxy-galaxy interaction or a mixture of both. Detailed comparisons between simulated and observed gas distributions and velocity fields allow us to identify the kind of interaction that the galaxy underwent and to determine the interaction parameters. In this way we are beginning to establish a temporal sequence of ram pressure stripping. We are now at the point where we can start to think about the gas physics of these interactions (evaporation, phase mixing, change of phase, star formation).

Baryons in Dark Matter Halos

5-9 October 2004

Novigrad, Croatia

${ }^{*}$ Speaker. 


\section{Introduction}

The Virgo cluster represents an ideal laboratory to study environmental effects on cluster galaxies. Virgo is the closest cluster in the northern hemisphere which can be observed in great detail $\left(1 \mathrm{kpc} \sim 12^{\prime \prime 1}\right)$. It is a dynamically young, spiral-rich cluster. Most of the spiral galaxies have entered the cluster only recently (within several Gyr, Tully \& Shaya 1984). It is known since the eighties that galaxies in nearby clusters are different from fi eld galaxies of the same size and morphological type: they are redder (Kennicutt 1983), have less HI (Chamaraux et al. 1980, Giovanelli \& Haynes 1983) and have truncated Hi disks (Warmels 1988, Cayatte et al. 1990). The cluster environment thus changes the properties of the cluster members. One can distinguish two classes of interactions between the cluster and a galaxy:

- Gravitational interactions: with the cluster potential (Byrd \& Valtonen 1990, Valluri 1993) and with other cluster galaxies (galaxy "harassment”; Moore et al. 1996, 1998).

- Interaction of the interstellar medium and the intracluster medium (ram pressure stripping; Gunn \& Gott 1972).

The observations of the last years gave us new insights into the evolution of Virgo cluster galaxies.

\section{The giant elliptical galaxy M86}

Galaxy clusters are the location of the largest diffuse radio emission sources in the local Universe. A very hot (several $\left.10^{7} \mathrm{~K}\right)$ and tenuous $\left(n \sim 10^{-4} \mathrm{~cm}^{-3}\right)$ gas pervades the whole cluster. This gas can be directly observed in X-rays (see e.g. Ebeling et al. 1996). The existence of a cluster-wide diffuse radio emission indicates that there are additional, important components of the intracluster medium (ICM): large-scale magnetic fi elds and relativistic electrons. These diffuse, extended radio sources are rare and there is strong evidence that they are related to cluster mergers (Giovannini \& Feretti 2002). We used the Effelsberg 100-m telescope at $1.4 \mathrm{GHz}$ to map a $10^{\circ} \times 10^{\circ}$ fi eld centered on Virgo A in the equatorial coordinate system (Vollmer et al. 2004a). When conducting a search for a presumably weak radio halo from the Virgo cluster, the presence of the quite bright radio source Virgo A (3C274, M87) must be taken into account. At $1.4 \mathrm{GHz}$ the peak flux density is about $150 \mathrm{Jy}$ when measured with the Effelsberg telescope. Such a strong signal produces antenna sidelobes within the area of the cluster what must be adequately taken into account. Another complication arises from the North Polar Spur (NPS), an intense Galactic radio shell, which is likely the old remnant from a nearby supernova explosion (Fig. 1). The analysis of these data showed that the Virgo cluster does not host a bright, large-scale radio halo. However, we cannot exclude the existence of a weak radio halo centered on M87 of a diameter smaller than $4^{\circ}$ $(\sim 1.2 \mathrm{Mpc})$ with a surface brightness smaller than one third of the radio halo in the Coma cluster. To our great surprise, we detect a low surface brightness radio halo with a flux density of $5 \pm 1.5 \mathrm{Jy}$ centered close to the elliptical galaxy M86 (Fig. 2). We believe that this radio halo is due to an interaction of the M86 intracluster medium with the Virgo intracluster medium. In this case M86 is falling into the Virgo cluster from behind. Since the timescale to build the radio halo is about

\footnotetext{
${ }^{1}$ We use a distance of $17 \mathrm{Mpc}$ to the Virgo cluster
} 


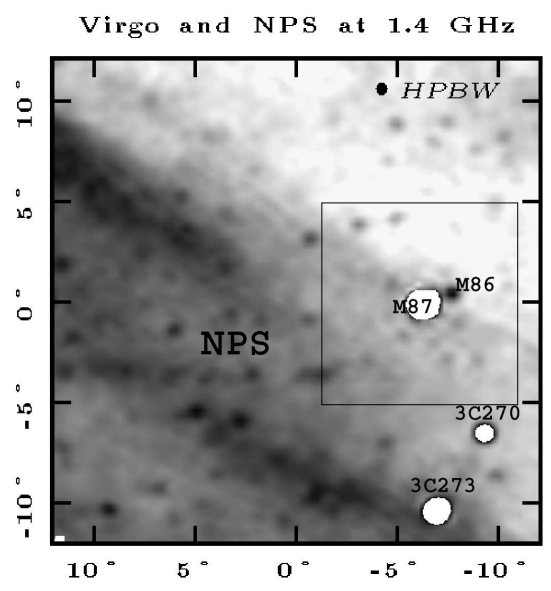

Figure 1: Left: section of the $1.4 \mathrm{GHz}$ Stockert continuum survey showing a part of the North Polar Spur close to the Virgo cluster with Virgo A or M87 at its center. The $10^{\circ} \times 10^{\circ}$ field observed with the Effelsberg telescope is indicated (from Vollmer et al. 2004a).

$1 \mathrm{Gyr}$, a possible scenario is that the interaction began at a distance of $\sim 1 \mathrm{Mpc}$ to $2 \mathrm{Mpc}$ from the cluster center. This interaction decelerated the outer ICM of M86 whereas M86 itself continued its way into the Virgo cluster where it now meets Virgo ICM gas densities of several $10^{-4} \mathrm{~cm}^{-3}$ at a distance of 0.5 to $1 \mathrm{Mpc}$ from the cluster center responsible for the shock detected in the south-west of M86 with XMM (Finoguenov et al. 2004). However, Neilsen \& Tsvetanov (2000), using the method of surface brightness fluctuations, place M86 at 2.4 $\pm 1.4 \mathrm{Mpc}$ behind the cluster. Thus, a second scenario where the halo of M86 interacted with an X-ray fi lament proposed by Finoguenov et al. (2004) is not excluded.

\section{A multiwavelength view of the Virgo cluster spiral galaxy population}

To study the properties of Virgo spiral galaxies, three different tracers are generally used: $\mathrm{H} \alpha$ to trace the recent massive star formation, $\mathrm{CO}(1-0)$ as a tracer of the molecular gas and $\mathrm{HI}$ for the atomic gas. One can distinguish two different approaches using (i) integrated values or (ii) maps of spiral galaxies.

Gavazzi et al. (2002) analyzed H $\alpha$, NIR H band, $\mathrm{CO}(1-0)$ and Hi photometry of 369 cluster and isolated galaxies. They use the birth parameter, which is the ratio of the current to the integrated star formation (i.e. the total stellar mass) to characterize the star formation effi ciency of a galaxy. The birth parameter for an isolated galaxy can be predicted using a closed box evolution model (Boselli et al. 2001). The difference between the observed and the predicted birth rate of a galaxy of a given $\mathrm{H}$ band luminosity indicates if its current star formation is enhanced or suppressed. The total gas content $(\mathrm{CO}+\mathrm{HI})$ of a cluster galaxy can be compared to that of an isolated galaxy of the same Hubble type. In this way one can defi ne a gas defi ciency parameter which is the logarithmic difference between the gas mass of isolated galaxies and the observed gas mass. Using these parameters Gavazzi et al. (2002) found that (i) massive galaxies form stars at a lower rate than their 

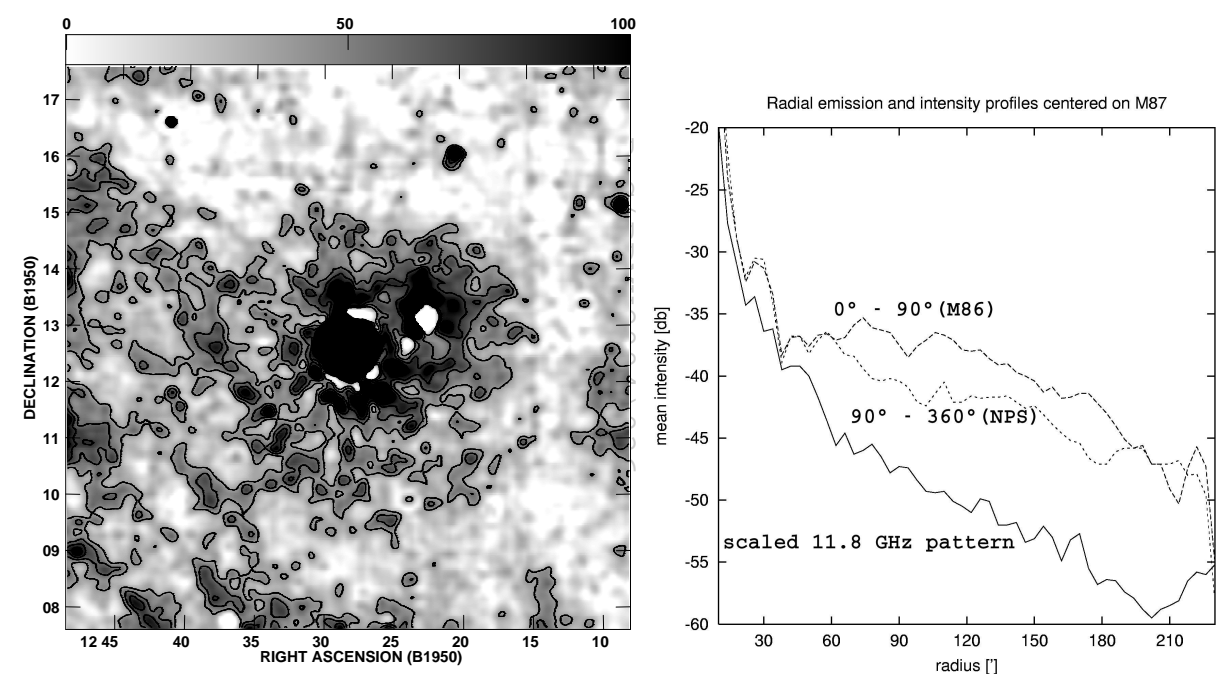

Figure 2: Left: Effelsberg 1.4 GHz total power map where the point sources are subtracted, the sidelobes are removed, and the large-scale structure is added. There is diffuse, large-scale emission around M86 which is located west of the bright central source M87. Right: radial intensity profiles measured at $11.8 \mathrm{GHz}$ using the EUTELSAT satellite and scaled to $1.4 \mathrm{GHz}$ in comparison with mean radial intensities measured in the sectors $0^{\circ}-90^{\circ}$ and $90^{\circ}-360^{\circ}$ (counterclockwise from north). We clearly detect a halo excess emission around M86 (from Vollmer et al. 2004a).

less massive counterparts and (ii) that gas defi cient galaxies have suppressed star formation rates (Fig. 3). The scatter of the corresponding relations is quite large. Since cluster galaxies are not CO defi cient (Kenney \& Young 1989, Casoli et al. 1991, Boselli et al. 1997), the gas defi ciency reflects the lack of HI.

High-resolution radio observations have beamsizes of the order of $20^{\prime \prime}$ (IRAM 30m CO(1-0), VLA C confi guration HI), i.e. $\sim 1.6 \mathrm{kpc}$. Thus, we can study the gas content of Virgo spiral galaxies in detail. The HI gas distribution of the 20 brightest Virgo spirals was studied by Cayatte et al. (1990). They showed that the Hi defi ciency is mainly due to the truncation of the gas disks. In general, the inner Hi surface densities of these galaxies are comparable to those of isolated galaxies. Only 3 out of 24 galaxies show a signifi cantly decreased inner Hi surface density.

Koopmann \& Kenney (2004a and b) compared $\mathrm{H} \alpha$ radial profi les of 29 isolated and 55 Virgo cluster galaxies. They divided the Virgo population into several classes: (i) normal, (ii) anemic (low, but measurable $\mathrm{H} \alpha$ all over the disk), (iii) enhanced and (iv) truncated H $\alpha$ disks. The class of truncated galaxies is subdivided into truncated/normal (Fig. 4), truncated/anemic and truncated/compact/enhanced. They found only a small fraction of anemic galaxies in both, the field and the cluster sample. Moreover, the majority of Virgo spiral galaxies have truncated H $\alpha$ disks. These galaxies are strongly concentrated in the cluster core.

As a conclusion, Virgo spiral galaxies have truncated gas disks making them gas defi cient. These galaxies also show truncated star formation within their disks. Within the truncation radius most of the disks show normal gas surface densities and star formation rates. Among the above cited interactions between a galaxy and its cluster environment, only ram pressure stripping can naturally account for these observational facts. 

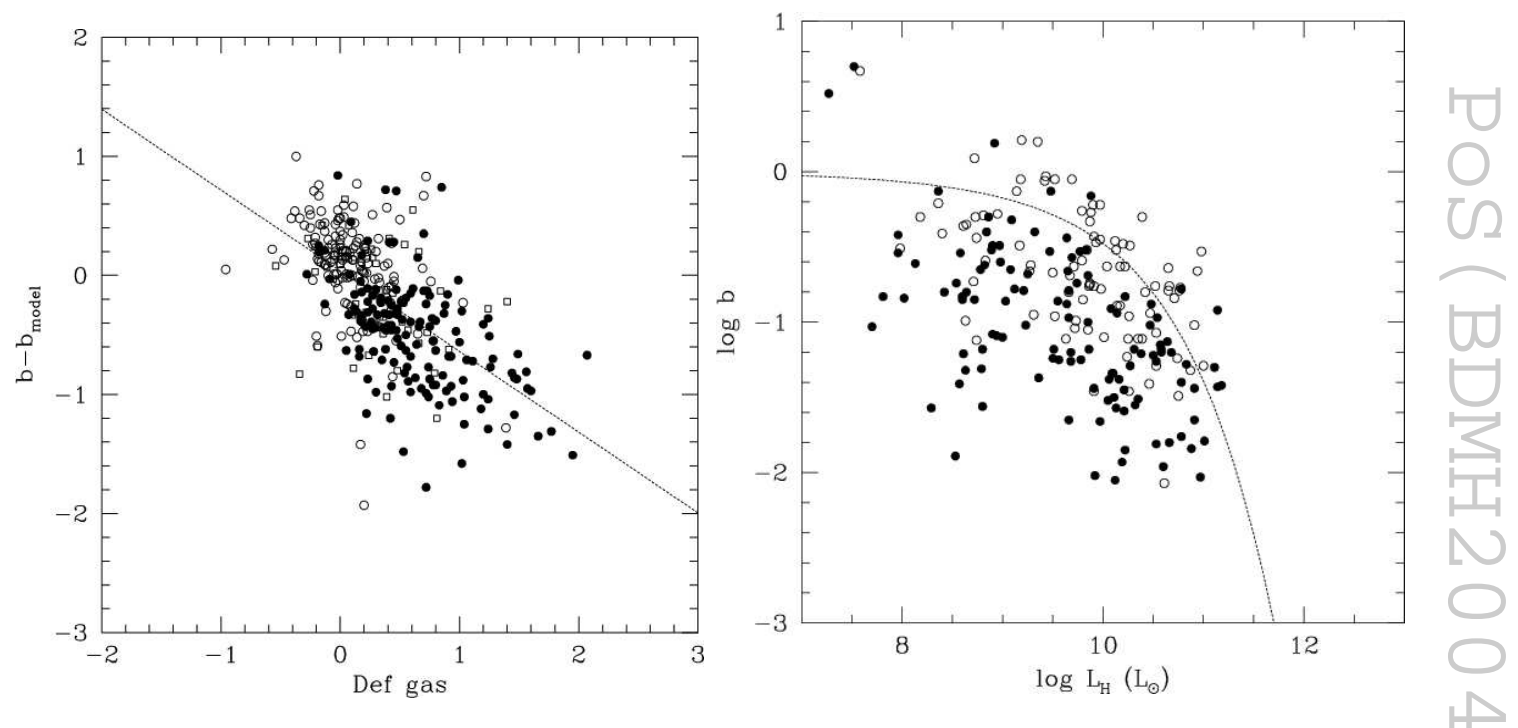

Figure 3: Left: difference between the observed and estimated birth rate parameter as a function of the gas deficiency. Gas deficient galaxy have suppressed star formation rates. Right: birth parameter as a function of the $\mathrm{H}$ band luminosity for gas normal (empty circles) and gas deficient (full circles) galaxies (from Gavazzi et al. 2002).
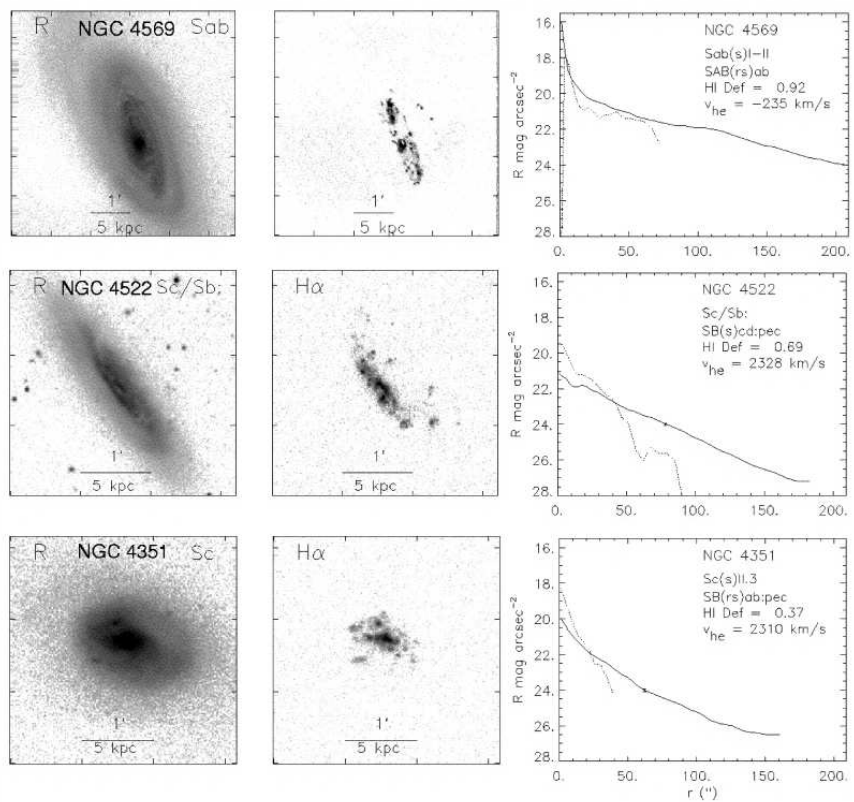
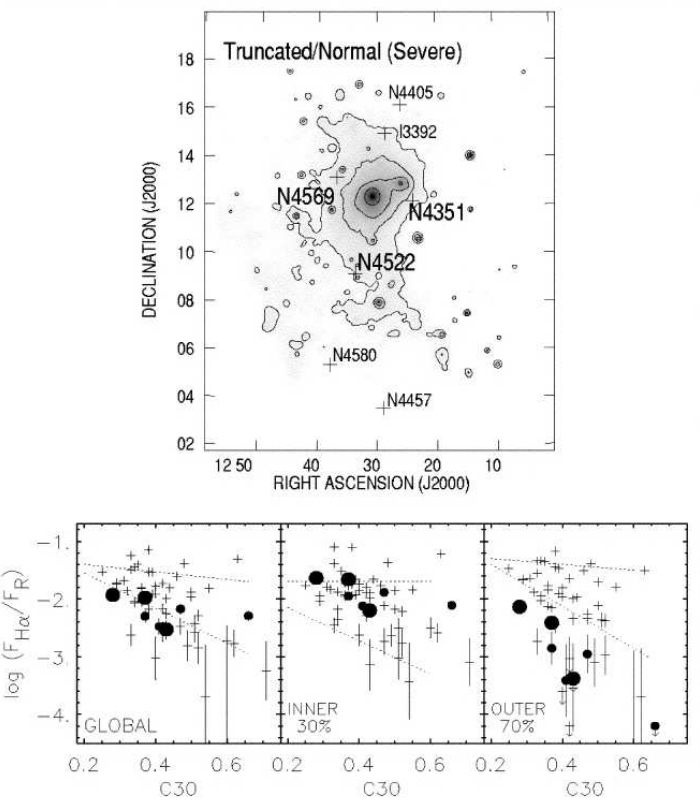

Figure 4: Three Virgo spiral galaxies with truncated H $\alpha$ disks (from Koopmann \& Kenney 2004b). 


\section{Towards a time sequence for ram pressure stripping in the Virgo cluster}

In a series of papers we used a numerical $\mathrm{N}$-body code, which includes a collisional and a non-collisional component, to study the effects of ram pressure on Virgo cluster spirals. As a fi rst approach we simulated galaxy orbits in the gravitational potential of the Virgo cluster to calculate the temporal ram pressure profi le (Vollmer et al. 2001). Since the distribution of the intracluster medium of the Virgo cluster is highly peaked on M87, very eccentric orbits are needed to strip Virgo galaxies signifi cantly. We then made N-body simulations of galaxies on different orbits, varying the peak ram pressure, the width of the ram pressure profi le and the inclination between the galaxy's disk and the orbital plane. With these simulations we were able to reproduce the HI observations of Cayatte et al. (1990). The main conclusion is that even with different, impulsive stripping events, the resulting HI distributions are truncated disks with normal, inner HI surface densities with respect to isolated galaxies. This work represents the normalization of the effects of ram pressure in the numerical code. We then applied our code to HI and CO observations of 6 Virgo spiral galaxies. Whenever we observed a galaxy deeper in HI than before, new and unexpected features were detected. Observationally, we find three different classes of galaxies showing: (i) extraplanar, high surface brightness gas (NGC 4522 Kenney et al. 2004, Vollmer et al. 2004b; NGC 4438 Vollmer et al. 2005 in prep.), (ii) extraplanar, very low surface brightness gas (NGC 4388 Vollmer \& Huchtmeier 2003) and (iii) perturbed, low surface brightness arms (NGC 4548 Vollmer et al. 1999; NGC 4569 Vollmer et al. 2004c, NGC 4654 Vollmer 2003; NGC 4254 Vollmer et al. 2005 in prep.). Our sample is now large enough to establish a fi rst time sequence for ram pressure stripping (Fig. 5 ). When ram pressure is maximum, high surface density gas is pushed out of the galaxy disk (NGC 4522, NGC 4438). This gas can be observed in Hi and $\mathrm{CO}$. When pushed to larger distances from the galactic disk the gas is expanding and/or evaporating. After one rotation period ( $\sim 100 \mathrm{Myr}$ ) only a small fraction of the gas can still be observed in HI (NGC 4388). One or two rotation periods later the gas that has been violently pushed to smaller galactic radii is resettling forming perturbed low surface density arms (NGC 4569, NGC 4548). NGC 4654 and NGC 4254 also show low surface density arms which are most probably due to the mixture of a tidal interaction, ram pressure and evaporation. Since our galaxy sample is still small, we need more observations and modeling to confi rm this time sequence.

\section{Polarized radio continuum emission - an ideal complementary tool for interaction diagnostics}

Observations of the polarized radio continuum radiation can give important and complementary information on the gas dynamics of perturbed Virgo spiral galaxies. This radiation traces the ordered large scale $(\sim 1 \mathrm{kpc})$ magnetic fi eld in galactic ISM which is very sensitive to (i) compression and (ii) shear motion, which are both diffi cult to detect in radial velocity fi elds. In an exploratory paper (Otmianowska \& Vollmer 2003) we solved the induction equation using the velocity fi elds of a typical ram pressure stripping event (Vollmer et al. 2001) in order to calculate the evolution of the large scale magnetic fi eld. In a second step the polarized radio continuum emission was calculated and maps of its evolution were made that can be directly compared to observations. We improved the calculations and applied them to a fi rst individual case: NGC 4654. 
NGC 4438
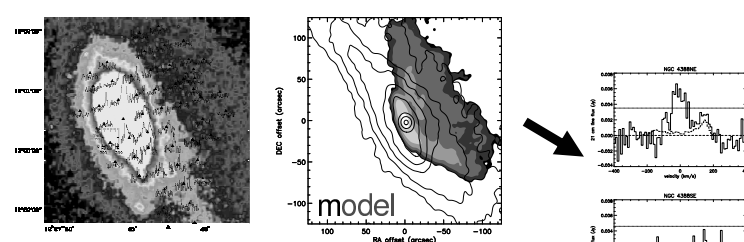

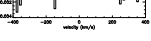
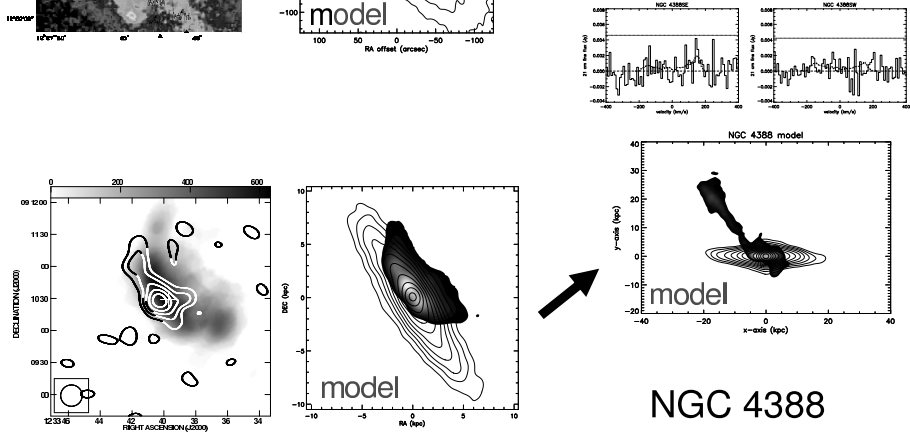

NGC 4388
NGC 4569
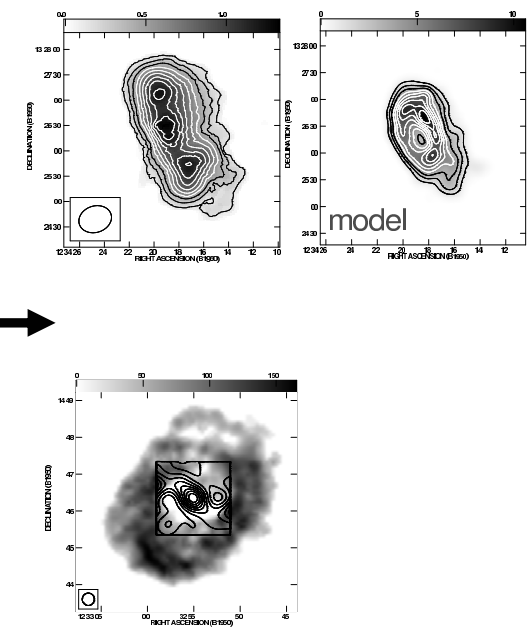

NGC 4548

NGC 4522

Figure 5: A first time sequence for ram pressure stripping in the Virgo cluster. Model images are marked as such.

Fig. 6 shows the observed (left panel) and modeled (right panel) HI, polarized radio continuum emission distributions and magnetic fi eld vectors. This fi rst example shows that polarized radio continuum emission is a powerful tool to detect interactions of spiral galaxies with the intracluster medium.

\section{References}

[1] Boselli, A., Gavazzi, G., Lequeux, J., Buat, V., Casoli, F., Dickey, J., \& Donas, J. 1997, A\&A, 327, 522

[2] Boselli, A., Gavazzi, G., Donas, J., \& Scodeggio, M. 2001, AJ, 121, 753

[3] Byrd, G. \& Valtonen, M. 1990, ApJ, 350, 89

[4] Casoli, F., Boissé, P., Combes, F., \& Dupraz, C. 1991, A\&A, 249, 359

[5] Cayatte, V., van Gorkom, J.H., Balkowski, C., \& Kotanyi C. 1990, AJ, 100, 604

[6] Chamaraux, P., Balkowski, C., \& Gérard, E. 1980, A\&A, 83, 38

[7] Ebeling, H., Voges ,W., Böhringer, H., et al. 1996, MNRAS, 281, 799

[8] Finoguenov, A., Pietsch, W., Aschenbach, B., \& Miniati, F. 2004, A\&A, 415, 415 

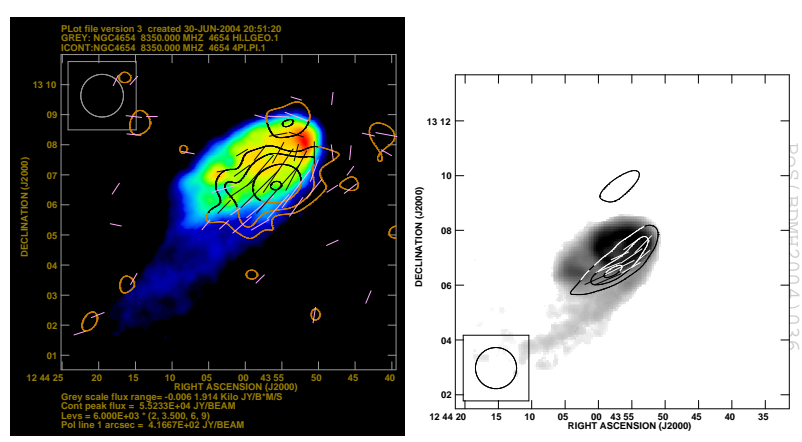

Figure 6: NGC 4654. Left: greyscale: HI distribution (VLA, Phookun \& Mundy 1995). Contours: polarized radio continuum emission (Effelsberg, Chyzy et al. in prep.). Lines: magnetic field vectors. Right: Modeled gas distribution (Vollmer 2003), radio continuum emission and magnetic field vectors (Soida et al. in prep.).

[9] Gavazzi, G., Boselli, A., Pedotti, P., Gallazzi A., \& Carrasco, L. 2002, A\&A, 396, 449

[10] Giovanelli, R. \& Haynes, M.P. 1985, ApJ, 292, 404

[11] Giovannini, G. \& Feretti, L. 2002, in: Merging Processes in Galaxy Clusters, Ed. L. Feretti, I. M. Gioia, \& G. Giovannini, Astrophysics and Space Science Library, Vol. 272 (Dordrecht: Kluwer) p. 197

[12] Gunn, J.E. \& Gott, J.R. 1972, ApJ, 176, 1

[13] Kenney, J.D. \& Young, J.S. 1989, Apj, 344, 171

[14] Kenney, Jeffrey D.P., van Gorkom, J.H., \& Vollmer, B. 2004, AJ, 127, 3361

[15] Kennicutt, R.C.Jr. 1983, AJ, 88, 483

[16] Koopmann, R. \& Kenney, J.D.P. 2004a, ApJ, 613, 851

[17] Koopmann, R. \& Kenney, J.D.P. 2004a, ApJ, 613, 866

[18] Moore, B., Katz, N., Lake, G., Dressler, A., \& Oemler A. 1996, Nature, 379, 613

[19] Moore, B., Lake, G., \& Katz, N. 1998, ApJ, 495, 139

[20] Neilson, E. H., \& Tsvetanov, Z. I. 2000, ApJ, 536, 255

[21] Otmianowska-Mazur, K. \& Vollmer, B. 2002, A\&A, 402, 879

[22] Tully, R.B. \& Shaya, E.J. 1984, ApJ, 281, 31

[23] Valluri, M. 1993 ApJ, 408, 57

[24] Vollmer, B., Cayatte, V., Boselli, A., Balkowski, C., \& Duschl, W.J. 1999, A\&A, 349, 411

[25] Vollmer, B., Cayatte, V., Balkowski, C., \& Duschl, W.J. 2001, ApJ, 561, 708

[26] Vollmer, B. 2003, A\&A, 398, 525

[27] Vollmer, B. \& Huchtmeier, W. 2003, A\&A, 406, 427

[28] Vollmer, B., Reich, W., \& Wielebinski, R. 2004a, A\&A, 423, 57

[29] Vollmer, B., Beck, R., Kenney, J.D.P., \& van Gorkom, J.H. 2004b, AJ, 127, 3375

[30] Vollmer, B., Balkowski, C., Cayatte, V., van Driel, W., \& Huchtmeier, W. 2004c, A\&A, 419, 35

[31] Warmels, R.H. 1988, A\&AS, 72, 19 